\title{
BOUNDARY BEHAVIOUR OF ANALYTIC FUNCTIONS IN SPACES OF DIRICHLET TYPE
}

\author{
DANIEL GIRELA AND JOSÉ ÁNGEL PELÁEZ
}

Received 24 June 2005; Revised 11 October 2005; Accepted 8 November 2005

For $0<p<\infty$ and $\alpha>-1$, we let $\mathscr{D}_{\alpha}^{p}$ be the space of all analytic functions $f$ in $\mathbb{D}=\{z \in$ $\mathbb{C}:|z|<1\}$ such that $f^{\prime}$ belongs to the weighted Bergman space $A_{\alpha}^{p}$. We obtain a number of sharp results concerning the existence of tangential limits for functions in the spaces $\mathscr{D}_{\alpha}^{p}$. We also study the size of the exceptional set $E(f)=\left\{e^{i \theta} \in \partial \mathbb{D}: V(f, \theta)=\infty\right\}$, where $V(f, \theta)$ denotes the radial variation of $f$ along the radius $\left[0, e^{i \theta}\right)$, for functions $f \in \mathscr{D}_{\alpha}^{p}$.

Copyright (C 2006 D. Girela and J. Á. Peláez. This is an open access article distributed under the Creative Commons Attribution License, which permits unrestricted use, distribution, and reproduction in any medium, provided the original work is properly cited.

\section{Introduction and main results}

Let $\mathbb{D}$ denote the open unit disk of the complex plane $\mathbb{C}$. If $0<r<1$ and $f$ is an analytic function in $\mathbb{D}$ (abbreviated $f \in \operatorname{H} o l(\mathbb{D})$ ), we set

$$
\begin{gathered}
M_{p}(r, f)=\left(\frac{1}{2 \pi} \int_{0}^{2 \pi}\left|f\left(r e^{\mathrm{it}}\right)\right|^{p} d t\right)^{1 / p}, \quad I_{p}(r, f)=M_{p}^{p}(r, f), \quad 0<p<\infty, \\
M_{\infty}(r, f)=\sup _{0 \leq t \leq 2 \pi}\left|f\left(r e^{\mathrm{it}}\right)\right| .
\end{gathered}
$$

For $0<p \leq \infty$, the Hardy space $H^{p}$ consists of those functions $f \in \operatorname{Hol}(\mathbb{D})$ for which $\|f\|_{H^{p}} \stackrel{\text { def }}{=} \sup _{0<r<1} M_{p}(r, f)<\infty$. We refer to [10] for the theory of Hardy spaces.

The weighted Bergman space $A_{\alpha}^{p}(0<p<\infty, \alpha>-1)$ is the space of all functions $f \in$ Hol(D) such that

$$
\|f\|_{A_{\alpha}^{p}} \stackrel{\text { def }}{=}\left(\int_{\mathbb{D}}(1-|z|)^{\alpha}|f(z)|^{p} d A(z)\right)^{1 / p}<\infty,
$$

where $d A(z)=(1 / \pi) d x d y$ denotes the normalized Lebesgue area measure in $\mathbb{D}$. We mention $[11,16]$ as general references for the theory of Bergman spaces. 
We will write $\mathscr{D}_{\alpha}^{p}(0<p<\infty, \alpha>-1)$ for the space of all functions $f \in \operatorname{Hol}_{(\mathbb{D})}$ such that $\int_{\mathbb{D}}(1-|z|)^{\alpha}\left|f^{\prime}(z)\right|^{p} d A(z)<\infty$. In other words,

$$
f \in \mathscr{D}_{\alpha}^{p} \Longleftrightarrow f^{\prime} \in A_{\alpha}^{p}
$$

If $p<\alpha+1$, it is well known that $\mathscr{D}_{\alpha}^{p}=A_{\alpha-p}^{p}$ with equivalence of norms (see [12, Theorem 6]). If $p>1$ and $\alpha=p-2$, we are considering the Besov spaces $\mathscr{B}^{p}$ which have been extensively studied in $[3,9,29]$. Specially relevant is the space $\mathscr{B}^{2}=\mathscr{D}_{0}^{2}$, which coincides with the classical Dirichlet space $\mathscr{D}$.

The space $\mathscr{D}_{\alpha}^{p}$ is said to be a Dirichlet space if $p \geq \alpha+1$. Specially interesting are the spaces in the "limit case" $p=\alpha+1$, that is, the spaces $\mathscr{D}_{p-1}^{p}, 0<p<\infty$. These spaces are closely related to Hardy spaces. Indeed, a direct calculation with Taylor coefficients gives that $H^{2}=\mathscr{D}_{1}^{2}$. Furthermore, we have

$$
\begin{array}{ll}
H^{p} \subset \mathscr{D}_{p-1}^{p}, & 2 \leq p<\infty, \\
\mathscr{D}_{p-1}^{p} \subset H^{p}, & 0<p \leq 2 .
\end{array}
$$

The relation (1.4) is a classical result of Littlewood and Paley [21], and (1.5) can be found in [28]. A good number of results on the spaces $\mathscr{D}_{p-1}^{p}$ have been recently obtained in $[4,13-15,28]$. We remark that the spaces $\mathscr{D}_{p-1}^{p}$ are not nested. Actually, it is easy to see that if $p \neq q$, then there is no relation of inclusion between $\mathscr{D}_{p-1}^{p}$ and $\mathscr{D}_{q-1}^{q}$.

Fatou's theorem asserts that if $0<p \leq \infty$ and $f \in H^{p}$, then $f$ has a finite nontangential limit $f\left(e^{i \theta}\right)$ for a.e. $e^{i \theta} \in \partial \mathbb{D}$. Bearing in mind (1.5), we see that this is true if $f \in \mathscr{D}_{p-1}^{p}$ and $0<p \leq 2$. In view of (1.4), it is natural to ask whether or not Fatou's theorem remains true for the spaces $\mathscr{D}_{p-1}^{p}, 2<p<\infty$. The answer to this question is negative. Indeed, [15, Theorem 3.5] asserts that if $2<p<\infty$, then there exists a function $f \in \mathscr{D}_{p-1}^{p}$ such that

$$
\lim _{r \rightarrow 1^{-}} \frac{\left|f\left(r e^{\mathrm{it}}\right)\right|}{(\log 1 /(1-r))^{1 / 2-1 / p}(\log \log 1 /(1-r))^{-1}}=\infty, \quad \text { for a.e. } e^{\text {it }} \in \partial \mathbb{D} \text {. }
$$

This function has a nontangential limit almost nowhere in $\partial D$.

Fatou's theorem is best possible for Hardy spaces in the sense that it cannot be extended further to give the existence of "tangential limits." Indeed, Lohwater and Piranian [22] (see also [8, page 43], [20, 31], and [32, Volume I, page 280] for some related results) proved that if $\gamma_{0}$ is a Jordan curve, internally tangent to $\partial \mathbb{D}$ at $z=1$, and having no other point in common with $\partial \mathbb{D}$, and $\gamma_{\theta}(\theta \in \mathbb{R})$ denotes the rotation of $\gamma_{0}$ through an angle $\theta$ around the origin, then there exists a function $f \in H^{\infty}$ such that, for every $\theta \in \mathbb{R}, f$ does not approach a limit as $z \rightarrow e^{i \theta}$ along $\gamma_{\theta}$.

In spite of this, a number of "tangential Fatou's theorems" have been proved for certain spaces of Dirichlet type. 
For $A>0, \gamma \geq 1$, and $\xi \in \partial \mathbb{D}$, we define

$$
R(A, \gamma, \xi)=\left\{z \in \mathbb{D}:|1-\bar{\xi} z|^{\gamma} \leq A(1-|z|)\right\}
$$

When $\gamma=1$ and $A>1$, the region $R(A, \gamma, \xi)$ is basically a Stolz angle. When $\gamma>1, R(A, \gamma, \xi)$ is a region contained in $\mathbb{D}$ which touches $\partial \mathbb{D}$ at $\xi$ tangentially. As $\gamma$ increases, the degree of tangency increases.

We define also, for $A>1$ and $\beta>0$,

$$
\begin{gathered}
R_{\exp }(A, \beta, \xi)=\left\{z \in \mathbb{D}: \exp \left(-|1-\bar{\xi} z|^{-\beta}\right) \leq \frac{(1-|z|)}{A}\right\}, \\
R_{\log }(A, \beta, \xi)=\left\{z \in \mathbb{D}:|1-\bar{\xi} z| \leq A(1-|z|)\left(\log \frac{2}{1-|z|}\right)^{\beta}\right\} .
\end{gathered}
$$

As $\beta$ increases, the degree of tangency increases in both types of tangential regions.

If $f \in \mathscr{H}$ ol( $(\mathbb{D})$, we say that $f$ has the $\gamma$-limit $L$ at $e^{i \theta}$, if $f(z) \rightarrow L$ as $z \rightarrow e^{i \theta}$ within $R(A, \gamma, \xi)$ for every $A$. Notice that saying that $f$ has the 1 -limit $L$ at $e^{i \theta}$ is the same as saying that $f$ has the nontangential limit $L$ at $e^{i \theta}$. Substituting the regions $R(A, \gamma, \xi)$ with the regions $R_{\exp }(A, \beta, \xi)$ and $R_{\log }(A, \beta, \xi)$, we have the notions of $\beta_{\exp }$-limits and $\beta_{\log }$-limits. We observe that these definitions of tangential limits are equivalent to those considered in $[2,7,23,26]$.

Among other results, Kinney [19] and Nagel, Rudin, and Shapiro [23] (see also [26]) proved the following.

(i) If $0<\alpha<1$ and $f \in D_{\alpha}^{2}$, then $f$ has a finite $\alpha^{-1}$-limit at a.e. $e^{i \theta} \in \partial \mathbb{D}$.

(ii) If $f \in D_{0}^{2}=\mathscr{D}$, then $f$ has a finite $1_{\text {exp }}$-limit almost everywhere.

In view of these results, it is natural to ask whether results of this kind can be proved for the spaces $\mathscr{D}_{\alpha}^{p}$ for other choices of $p$ and $\alpha$. We start with a negative result.

Theorem 1.1. (a) Suppose that $A>1$ and $\beta>1$. Then there exists a function $f \in$ $\bigcap_{1 \leq p<\infty} \mathscr{D}_{p-1}^{p}$ such that for almost every $e^{i \theta} \in \partial \mathbb{D}, f$ does not approach a limit as $z \rightarrow e^{i \theta}$ inside $R_{\log }\left(A, \beta, e^{i \theta}\right)$.

(b) Suppose that $A>0$ and $\gamma>1$. Then there exists a function $f \in \bigcap_{0<p<\infty} \mathscr{D}_{p-1}^{p}$ such that for almost every $e^{i \theta} \in \partial \mathbb{D}, f$ does not approach a limit as $z \rightarrow e^{i \theta}$ inside $R\left(A, \gamma, e^{i \theta}\right)$.

Next we turn our attention to the spaces $\mathscr{D}_{\alpha}^{p}$ with $1 \leq p \leq 2$ and $-1<\alpha \leq p-1$. We will prove the following theorem.

Theorem 1.2. (a) Suppose that $1 \leq p \leq 2, p-2<\alpha \leq p-1$, and $f \in \mathscr{D}_{\alpha}^{p}$. Then $f$ has an $(\alpha-p+2)^{-1}$-limit at a.e. $e^{i \theta} \in \partial \mathbb{D}$.

(b) Suppose that $1<p \leq 2$ and $f \in \mathscr{D}_{p-2}^{p}=\mathscr{B}_{P}$. Then $f$ has a $\left(p^{\prime}-1\right)_{\exp }$-limit at a.e. $e^{i \theta} \in \partial \mathbb{D}$.

Here and throughout the paper, if $p>1$, we write $p^{\prime}$ for the exponent conjugate of $\mathrm{p}$, $1 / p+1 / p^{\prime}=1$. 
We will prove that part (a) of Theorem 1.2 is sharp in the sense that the degree of potential tangency $(\alpha-p+2)^{-1}$ cannot be substituted by any larger one.

Theorem 1.3. Suppose that $1 \leq p \leq 2, p-2<\alpha \leq p-1, A>0$, and $\gamma>(\alpha-p+2)^{-1}$. Then there exists a function $f \in \mathscr{D}_{\alpha}^{p}$ such that for almost every $e^{i \theta} \in \partial \mathbb{D}, f$ does not approach a limit as $z \rightarrow e^{i \theta}$ inside $R\left(A, \gamma, e^{i \theta}\right)$.

Now we turn to questions related to radial variation of analytic functions. If $f \in$ $\operatorname{Hol}(\mathbb{D})$ and $\theta \in[-\pi, \pi)$, we define

$$
V(f, \theta) \stackrel{\text { def }}{=} \int_{0}^{1}\left|f^{\prime}\left(r e^{i \theta}\right)\right| d r
$$

Then $V(f, \theta)$ denotes the radial variation of $f$ along the radius $\left[0, e^{i \theta}\right)$, that is, the length of the image of this radius under the mapping $f$. We define the exceptional set $E(f)$ associated to $f$ as

$$
E(f)=\left\{e^{i \theta} \in \partial \mathbb{D}: V(f, \theta)=\infty\right\}
$$

It is clear that if $f$ has finite radial variation at $e^{i \theta}$, then $f$ has a finite radial limit at $e^{i \theta}$. Even though every $H^{p}$-function, $0<p \leq \infty$, has finite radial limits a.e., if we take $f \in \operatorname{Hol}(\mathbb{D})$ given by a power series with Hadamard gaps

$$
f(z)=\sum_{k=1}^{\infty} a_{k} z^{n_{k}} \quad \text { with } n_{k+1} \geq \lambda n_{k}, \forall k(\lambda>1),
$$

such that

$$
\sum_{k=1}^{\infty}\left|a_{k}\right|^{2}<\infty \text { but } \sum_{k=1}^{\infty}\left|a_{k}\right|=\infty,
$$

then $f \in \bigcap_{0<p<\infty} H^{p}$, but a result of Zygmund (see [30, Theorem 1, page 194]) shows that $V(f, \theta)=\infty$ for every $\theta \in[-\pi, \pi)$.

We will prove a positive result for $\mathscr{D}_{p-1}^{p}$-functions, $0<p \leq 1$.

Theorem 1.4. If $0<p \leq 1$ and $f \in \mathscr{D}_{p-1}^{p}$, then $E(f)$ has measure 0 .

We note that this result cannot be extended to $p>1$. Indeed, if we take $f$ given by a power series with Hadamard gaps as in (1.11) with $\sum_{k=1}^{\infty}\left|a_{k}\right| p<\infty$ and $\sum_{k=1}^{\infty}\left|a_{k}\right|=\infty$, we have that $f \in \mathscr{D}_{p-1}^{p}($ see $[15$, Proposition A $])$ and so $V(f, \theta)=\infty$ for every $\theta \in[-\pi, \pi)$.

On the other hand, we have the following well-known result of Beurling [5] for functions in $\mathscr{D}_{\alpha}^{2}$.

THeOREM 1.5. Let $f$ be an analytic function in $\mathbb{D}$.

(a) If $f \in \mathscr{D}$, then $E(f)$ has logarithmic capacity 0 .

(b) If $0<\alpha<1$ and $f \in \mathscr{D}_{\alpha}^{2}$, then $E(f)$ has $\alpha$-capacity 0 . 
See [17] for the definitions of logarithmic capacity and $\alpha$-capacity and [27] for an extension of Theorem 1.5.

We will prove the following result for other values of $p$.

Theorem 1.6. Suppose that $f \in \mathscr{D}_{\alpha}^{p}$.

(a) If $0<p \leq 1$ and $-1<\alpha<p-1$, then $E(f)$ has Lebesgue measure 0 .

(b) If $1<p<2$ and $p-2<\alpha<p-1$, then $E(f)$ has Lebesgue measure 0 .

(c) If $1<p \leq 2$ and $\alpha=p-2$, then $E(f)$ has logarithmic capacity 0 .

(d) If $2<p<\infty$ and $p-1>\alpha \geq p / 2-1$, then $E(f)$ has $\beta$-capacity 0 for all $\beta>2 / p(1+$ $\alpha)-1$.

(e) If $2<p<\infty$ and $\alpha<p / 2-1$, then $E(f)$ has logarithmic capacity 0 .

\section{On the membership of Blaschke products in spaces of Dirichlet type}

We remark that $H^{\infty} \not \subset \mathscr{D}_{\alpha}^{p}$, if $0<p<\infty$ and $-1<\alpha<p-1$ (see, e.g., [13, Section 3] for explicit examples). Clearly, (1.4) gives that $H^{\infty} \subset \mathscr{D}_{p-1}^{p}$, if $2 \leq p<\infty$. However, this does not remain true for $0<p<2$. Indeed, Vinogradov [28, pages 3822-3823] has shown that there exist Blaschke products $B$ which do not belong to $\bigcup_{0<p<2} \mathscr{D}_{p-1}^{p}$. In this section, we will find a number of sufficient conditions for the membership of a Blaschke product in some of the spaces $\mathscr{D}_{\alpha}^{p}$. These results will be basic in the proofs of Theorems 1.1 and 1.3.

We recall that if a sequence of points $\left\{a_{n}\right\}$ in $\mathbb{D}$ satisfies the Blaschke condition $\sum_{n=1}^{\infty}(1-$ $\left.\left|a_{n}\right|\right)<\infty$, the corresponding Blaschke product $B$ is defined as

$$
B(z)=\prod_{n=1}^{\infty} \frac{\left|a_{n}\right|}{a_{n}} \frac{a_{n}-z}{1-\overline{a_{n}} z} .
$$

Such a product is analytic in $\mathbb{D}$, bounded by one, and with nontangential limits of modulus one almost everywhere on the unit circle. We start obtaining sufficient conditions for the membership of a Blaschke product in the spaces $\mathscr{D}_{p-1}^{p}$, improving the first part of [28, Lemma 2.11].

LeMmA 2.1. Let B be a Blaschke product with sequence of zeros $\left\{a_{n}\right\}$.

(a) If $\left\{a_{n}\right\}$ satisfies

$$
\sum_{n=1}^{\infty}\left(1-\left|a_{n}\right|\right) \log \left(\frac{1}{1-\left|a_{n}\right|}\right)<\infty
$$

then $B \in \bigcap_{1 \leq p<\infty} \mathscr{D}_{p-1}^{p}$.

(b) If there exists $q \in(0,1)$ such that

$$
\sum_{n=1}^{\infty}\left(1-\left|a_{n}\right|\right)^{q}<\infty
$$

then $B \in \bigcap_{0<p<\infty} \mathscr{D}_{p-1}^{p}$. 
Proof. A result of Rudin's (see [25, Theorem I]) shows that (2.2) implies that $B \in \mathscr{D}_{0}^{1}$. Then (a) follows from the Cauchy estimate $\left|B^{\prime}(z)\right| \leq 1 /(1-|z|)$.

We turn now to part (b). Suppose that $\left\{a_{n}\right\}$ satisfies (2.3) for a certain $q \in(0,1)$. Assume for now that $p \in(0,1]$. Using [18, Theorem 3.1], we see that $B^{\prime} \in A^{2-q}$. Using this, Hölder's inequality with exponents $(2-q) / p$ and $(2-q) /(2-q-p)$, and the fact that $(2-q)(1-p) /(2-q-p)<1$, we obtain

$$
\begin{aligned}
& \int_{\mathbb{D}}\left|B^{\prime}(z)\right|^{p}\left(1-|z|^{2}\right)^{p-1} d A(z) \\
& \quad \leq\left(\int_{\mathbb{D}}\left|B^{\prime}(z)\right|^{2-q} d A(z)\right)^{p /(2-q)}\left(\int_{\mathbb{D}}\left(1-|z|^{2}\right)^{(2-q)(p-1) /(2-q-p)} d A(z)\right)^{(2-q-p) /(2-q)} \\
& \quad<\infty .
\end{aligned}
$$

Hence, we have shown that $B \in \mathscr{D}_{p-1}^{p}$, for all $p \in(0,1]$. Using the Cauchy estimate again, we obtain that $B \in \mathscr{D}_{p-1}^{p}$ for all $p \in(0, \infty)$, as desired.

We next give a simplified proof of a result that essentially is Theorem 3.1(i) for $\beta=1$ and $p \geq 1$ in [18].

Lemma 2.2. Let $p$ and $\alpha$ be such that $p \geq 1$ and $p-2<\alpha<p-1$. If $B$ is a Blaschke product whose sequence of zeros $\left\{a_{n}\right\}$ satisfies

$$
\sum_{n=1}^{\infty}\left(1-\left|a_{n}\right|\right)^{\alpha+2-p}<\infty
$$

then $B \in \mathscr{D}_{\alpha}^{p}$.

Proof. We will use the notation and terminology of [1, pages 332-333].

Let $p, \alpha$, and $B$ be as in the statement. Notice that $0<\alpha+2-p<1$, and then, using [24, Theorem 1], we deduce that $B^{\prime} \in \mathrm{B}^{1 /(\alpha-p+3)}$ or, equivalently, $B \in \mathscr{D}_{\alpha-p+1}^{1}$. Then as in the proof of Lemma 2.1, the Cauchy estimate implies $B \in \mathscr{D}_{\alpha}^{p}$ since $p-1 \geq 0$.

\section{Tangential limits for $\mathscr{D}_{\alpha}^{p}$-functions}

Proof of Theorem 1.1(a). We are going to use an argument which is similar to the one used in the proof of [32, Volume I, Chapter VII, Theorem 7.44].

Take $M$ with $1<M<A$ and let $C_{\theta}$ be the boundary of $R_{\log }\left(M, \beta, e^{i \theta}\right)(\theta \in[0,2 \pi))$. For all sufficiently large $n$, let $l_{n}$ denote the length of the arc of the circle $|z|=1-1 / n$ which lies in $R_{\log }(M, \beta, 1)$ and let $m_{n}=E\left[2 \pi / l_{n}\right]+1$, where, for $x \in \mathbb{R}, E[x]$ denotes the greatest integer that is smaller than or equal to $x$. Let $S_{n}=\left\{z_{n, 1}, z_{n, 2}, \ldots, z_{n, m_{n}}\right\}$ be any collection of $m_{n}$ points equally spaced on $|z|=1-1 / n$. Since the circular distance between any two consecutive points of $S_{n}$ is smaller than $l_{n}$, for every $\theta$ the set $R_{\log }\left(M, \beta, e^{i \theta}\right)$ contains a point of $S_{n}$. 
We define

$$
\sigma_{n}=\sum_{k=1}^{m_{n}}\left(1-\left|z_{n, k}\right|\right) \log \left(\frac{1}{1-\left|z_{n, k}\right|}\right)=\frac{m_{n} \log (n)}{n}
$$

Notice that $l_{n} \asymp(1 / n) \log ^{\beta} n$. Then it is easy to see that there exists a positive constant $\mathrm{C}$ (which does not depend on $n$ ) such that

$$
\sigma_{n}=\frac{m_{n} \log (n)}{n} \leq \frac{\left(1+2 \pi / l_{n}\right) \log (n)}{n} \leq C \frac{\log (n)}{n l_{n}} \leq C \frac{1}{\log ^{\beta-1} n} \longrightarrow 0, \quad \text { as } n \longrightarrow \infty .
$$

Let us take then an increasing sequence $n_{k}$ satisfying that $\sum_{k=1}^{\infty} \sigma_{n_{k}}<\infty$ and let $B$ be the Blaschke product with zeros at the points of $\bigcup_{k=1}^{\infty} S_{n_{k}}$. By part (a) of Lemma 2.1, $B \in \bigcap_{1 \leq p<\infty} \mathscr{D}_{p-1}^{p}$. Notice that for each $\theta \in \mathbb{R}, B$ has infinitely many zeros in the set $R_{\log }\left(M, \beta, e^{i \theta}\right)$. Thus for every $\theta$, the limit of $B(z)$ as $z \rightarrow e^{i \theta}$ inside of $R_{\log }\left(M, \beta, e^{i \theta}\right)$ must be zero if it exists at all. Since the radial limit of $B$ has absolute value 1 a.e., it follows that for almost every $e^{i \theta} \in \partial \mathbb{D}$, the limit of $B(z)$ as $z \rightarrow e^{i \theta}$ inside of $R_{\log }\left(M, \beta, e^{i \theta}\right)$ does not exist.

Part(b) of Theorem 1.1 can be proved in a similar way using part (b) of Lemma 2.1. We omit the details.

Next we will obtain a representation formula for functions $f$ in the space $\mathscr{D}_{\alpha}^{p},-1<\alpha$, $1 \leq p \leq 2$, which will play a basic role in the proof of Theorem 1.2.

Theorem 3.1. Suppose that either $1 \leq p \leq 2$ and $-1<\alpha<p-1$ or $1<p \leq 2$ and $\alpha=$ $p-2$, and that $f \in \mathscr{D}_{\alpha}^{p}$. Then there exists a function $h\left(e^{i \theta}\right) \in L^{p}(\partial \mathbb{D})$ such that

$$
f(z)=\frac{1}{2 \pi} \int_{-\pi}^{\pi} \frac{h\left(e^{i \theta}\right)}{\left(1-e^{-i \theta} z\right)^{(\alpha+1) / p}} d \theta, \quad z \in \mathbb{D} .
$$

Proof. Let $p$ and $\alpha$ be as in the statement and $f(z)=\sum_{n=0}^{\infty} a_{n} z^{n} \in \mathscr{D}_{\alpha}^{p}$. Then $z f^{\prime}(z)=$ $\sum_{n=0}^{\infty} n a_{n} z^{n} \in A_{\alpha}^{p}$. Since $\mathscr{D}_{\alpha}^{p} \subset A_{\alpha}^{p}$, we also have that $f \in A_{\alpha}^{p}$. Then it follows that

$$
z f^{\prime}(z)+\frac{\alpha+1}{p} f(z)=\sum_{n=0}^{\infty}\left(n+\frac{\alpha+1}{p}\right) a_{n} z^{n} \in A_{\alpha}^{p}
$$

So using [6, Lemma 1.1] (see also [12, part (iii) of Theorem 5]) and [6, Corollary 3.5], we deduce that the fractional integral

$$
h(z) \stackrel{\text { def }}{=} \widetilde{I}^{(\alpha+1) / p}\left(z f^{\prime}(z)+\frac{\alpha+1}{p} f(z)\right)=\sum_{n=0}^{\infty}\left(n+\frac{\alpha+1}{p}\right) B\left(n+1, \frac{\alpha+1}{p}\right) a_{n} z^{n}
$$

belongs to $H^{p}$ since $p \leq 2$. Here $B(\cdot, \cdot)$ is the classical beta function. Note that

$$
B(u, v)=\frac{\Gamma(u) \Gamma(v)}{\Gamma(u+v)}
$$


8 Boundary behaviour

and recall that $\Gamma(s+1)=s \Gamma(s)$, for all $s \neq 0,-1, \ldots$ Then it is easy to see that

$$
h(z)=\sum_{n=0}^{\infty} \frac{n ! \Gamma((\alpha+1) / p)}{\Gamma(n+(\alpha+1) / p)} a_{n} z^{n}
$$

Then,

$$
h\left(e^{i \theta}\right)=\sum_{n=0}^{\infty} \frac{n ! \Gamma((\alpha+1) / p)}{\Gamma(n+(\alpha+1) / p)} a_{n} e^{i n \theta} \in L^{p}(\partial \mathbb{D}) .
$$

By the binomial theorem,

$$
\frac{1}{\left(1-e^{-i \theta} z\right)^{(\alpha+1) / p}}=\sum_{k=0}^{\infty} \frac{\Gamma(k+(\alpha+1) / p)}{k ! \Gamma((\alpha+1) / p)} e^{-i k \theta} z^{k} .
$$

Thus,

$$
\begin{aligned}
& \frac{1}{2 \pi} \int_{-\pi}^{\pi} \frac{h\left(e^{i \theta}\right)}{\left(1-e^{-i \theta} z\right)^{(\alpha+1) / p}} d t \\
& \quad=\frac{1}{2 \pi} \int_{-\pi}^{\pi}\left(\sum_{n=0}^{\infty} \frac{n ! \Gamma(\alpha+1 / p)}{\Gamma(n+(\alpha+1) / p)} a_{n} e^{i n \theta}\right)\left(\sum_{k=0}^{\infty} \frac{\Gamma(k+(\alpha+1) / p)}{k ! \Gamma(\alpha+1 / p)} e^{-i k \theta} z^{k}\right) d \theta \\
& \quad=\sum_{n=0}^{\infty} a_{n} z^{n}=f(z) .
\end{aligned}
$$

This finishes the proof.

Proof of Theorem 1.2. We need to consider three cases.

Case 1. $1 \leq p \leq 2$ and $\alpha=p-1$. Then $\mathscr{D}_{\alpha}^{p}=\mathscr{D}_{p-1}^{p} \subset H^{p}$ and the result in this case follows from Fatou's theorem for $H^{p}$.

Case 2. $1 \leq p \leq 2$ and $p-2<\alpha<p-1$. If $f \in \mathscr{D}_{\alpha}^{p}$, then, using Theorem 3.1, we have that there exists $h\left(e^{i \theta}\right) \in L^{p}(\partial \mathbb{D})$ such that

$$
f(z)=\frac{1}{2 \pi} \int_{-\pi}^{\pi} \frac{h\left(e^{i \theta}\right)}{\left(1-e^{-i \theta} z\right)^{(\alpha+1) / p}} d t=\frac{1}{2 \pi} \int_{-\pi}^{\pi} \frac{h\left(e^{i \theta}\right)}{\left(1-e^{-i \theta} z\right)^{1-(p-\alpha-1) / p}} d t .
$$

Notice that $p((p-\alpha-1) / p)<1$, so by [23, part (a) of Theorem A] we have that $\mathrm{f}$ has $(\alpha-p+2)^{-1}$-limit at a.e. $e^{i \theta} \in \partial \mathbb{D}$. 
Case 3. $1<p \leq 2$ and $\alpha=p-2$. Using again Theorem 3.1, we have that if $f \in \mathscr{D}_{\alpha}^{p}$, then there exists $h\left(e^{i \theta}\right) \in \iota^{p}(\partial \mathbb{D})$ such that

$$
f(z)=\frac{1}{2 \pi} \int_{-\pi}^{\pi} \frac{h\left(e^{i \theta}\right)}{\left(1-e^{-i \theta} z\right)^{1-1 / p}} d t .
$$

Using [23, part (b) of Theorem A], we deduce that $f$ has $\left(p^{\prime}-1\right)_{\exp }$-limit at a.e. $e^{i \theta} \in \partial \mathbb{D}$.

Theorem 1.3 can be proved arguing as in the proof of part (a) of Theorem 1.1, using Lemma 2.2 instead of Lemma 2.1. Again, we will omit the details.

\section{Radial variation of functions in the spaces $\mathscr{D}_{\alpha}^{p}$}

Proof of Theorem 1.4. Let $0<p<1$ and $f \in \mathscr{D}_{p-1}^{p}$. Set

$$
F_{f}=\left\{\theta \in[-\pi, \pi]: f \text { has a finite nontangential limit at } e^{i \theta}\right\} .
$$

By (1.5) and Fatou's theorem, $[-\pi, \pi] \backslash F_{f}$ has Lebesgue measure 0. On the other hand, Zygmund proved in [30, page 81$]$ that

$$
(1-r)\left|f^{\prime}\left(r e^{i \theta}\right)\right| \longrightarrow 0, \quad \text { as } r \longrightarrow 1^{-},
$$

for all $\theta \in F_{f}$. Consequently the set

$$
F_{f}^{*}=\left\{\theta \in[-\pi, \pi]:(1-r)\left|f^{\prime}\left(r e^{i \theta}\right)\right| \longrightarrow 0\right\}
$$

is such that $[-\pi, \pi] \backslash F_{f}^{*}$ has Lebesgue measure 0 . Since $f \in \mathscr{D}_{p-1}^{p}$, we deduce that the set

$$
T_{f}=\left\{\theta \in[-\pi, \pi]: \int_{0}^{1}(1-r)^{p-1}\left|f^{\prime}\left(r e^{i \theta}\right)\right|^{p} d r<\infty\right\}
$$

is such that $[-\pi, \pi] \backslash T_{f}$ has Lebesgue measure 0 . Thus, $[-\pi, \pi] \backslash\left(F_{f}^{*} \cap T_{f}\right)$ has Lebesgue measure 0 . Furthermore, if $\theta \in F_{f}^{*} \cap T_{f}$, there exists a positive constant $C_{\theta}$ such that

$$
V(f, \theta)=\int_{0}^{1}\left|f^{\prime}\left(r e^{i \theta}\right)\right|^{p}\left|f^{\prime}\left(r e^{i \theta}\right)\right|^{1-p} d r \leq C_{\theta} \int_{0}^{1}(1-r)^{p-1}\left|f^{\prime}\left(r e^{i \theta}\right)\right|^{p} d r<\infty .
$$

Proof of Theorem 1.6. Since

$$
\mathscr{D}_{\alpha}^{p} \subset \mathscr{D}_{\beta}^{p}, \quad-1<\alpha \leq \beta, 0<p<\infty,
$$

(a) follows from Theorem 1.4.

Suppose now that $1<p<2, p-2<\alpha<p-1$, and $f \in \mathscr{D}_{\alpha}^{p}$. Then the set

$$
T_{f}^{\alpha}=\left\{\theta \in[-\pi, \pi]: \int_{0}^{1}(1-r)^{\alpha}\left|f^{\prime}\left(r e^{i \theta}\right)\right|^{p} d r<\infty\right\}
$$


is such that $[-\pi, \pi] \backslash T_{f}^{\alpha}$ has Lebesgue measure 0 . Now, using Hölder's inequality, we see that there exists a positive constant $C_{\alpha, p}$ such that

$$
\begin{aligned}
V(f, \theta) & =\int_{0}^{1}(1-r)^{\alpha / p}\left|f^{\prime}\left(r e^{i \theta}\right)\right|(1-r)^{-\alpha / p} d r \\
& \leq\left(\int_{0}^{1}(1-r)^{\alpha}\left|f^{\prime}\left(r e^{i \theta}\right)\right|^{p} d r\right)^{1 / p}\left(\int_{0}^{1}(1-r)^{-p^{\prime} \alpha / p} d r\right)^{1 / p^{\prime}} \\
& \leq C_{\alpha, p}\left(\int_{0}^{1}(1-r)^{\alpha}\left|f^{\prime}\left(r e^{i \theta}\right)\right|^{p} d r\right)^{1 / p}<\infty,
\end{aligned}
$$

for all $\theta \in T_{f}^{\alpha}$. (We have used that $-p^{\prime} \alpha / p>-1$ since $\alpha<p-1$.) Thus, (b) is proved.

Part (c) follows from the well-known inclusion

$$
\mathscr{D}_{p-2}^{p}=\mathscr{B}^{p} \subset \mathscr{B}^{q}=\mathscr{D}_{q-2}^{q}, \quad 1<p<q<\infty,
$$

(see, e.g., [3, page 112]), Theorem 1.5, and the fact that $\mathscr{B}^{2}=\mathscr{D}$.

Finally, suppose that $2<p<\infty$ and $f \in \mathscr{D}_{\alpha}^{p}$. Using Hölder's inequality with exponents $p /(p-2)$ and $p / 2$, we have that

$$
\begin{aligned}
\int_{\mathbb{D}}(1-|z|)^{\beta}\left|f^{\prime}(z)\right|^{2} d A(z)= & \int_{\mathbb{D}}(1-|z|)^{\beta-2 \alpha / p}\left|f^{\prime}(z)\right|^{2}(1-|z|)^{2 \alpha / p} d A(z) \\
\leq & \left(\int_{\mathbb{D}}(1-|z|)^{(p \beta-2 \alpha) /(p-2)} d A(z)\right)^{(p-2) / p} \\
& \times\left(\int_{\mathbb{D}}(1-|z|)^{\alpha}\left|f^{\prime}(z)\right|^{p} d A(z)\right)^{2 / p}
\end{aligned}
$$

Letting $\beta=0$, we see that the condition $\alpha<p / 2-1$ implies that $f \in \mathscr{D}$. Hence, (e) follows from part (a) of Theorem 1.5. On the other hand, if $p-1>\alpha \geq p / 2-1$, then $\beta$ can be chosen so that $\beta>(2 / p)(1+\alpha)-1$ and $0<\beta<1$. Then (4.10) implies that $f \in \mathscr{D}_{\beta}^{2}$, and (d) follows from part (b) of Theorem 1.5.

\section{Acknowledgments}

We wish to thank the referee for his helpful remarks specially for those regarding the proof of Theorem 3.1. The authors are partially supported by grants from "El Ministerio de Educación y Ciencia, Spain,” and FEDER (MTM2004-00078 and MTM2004-21420-E) and by a grant from "La Junta de Andalucía" (FQM-210).

\section{References}

[1] P. R. Ahern, The mean modulus and the derivative of an inner function, Indiana University Mathematics Journal 28 (1979), no. 2, 311-347.

[2] P. R. Ahern and D. N. Clark, On inner functions with $\mathrm{H}^{p}$-derivative, The Michigan Mathematical Journal 21 (1974), 115-127. 
[3] J. Arazy, S. D. Fisher, and J. Peetre, Möbius invariant function spaces, Journal für die reine und angewandte Mathematik 363 (1985), 110-145.

[4] A. Baernstein II, D. Girela, and J. Á. Peláez, Univalent functions, Hardy spaces and spaces of Dirichlet type, Illinois Journal of Mathematics 48 (2004), no. 3, 837-859.

[5] A. Beurling, Ensembles exceptionnels, Acta Mathematica 72 (1939), 1-13.

[6] S. M. Buckley, P. Koskela, and D. Vukotić, Fractional integration, differentiation, and weighted Bergman spaces, Mathematical Proceedings of the Cambridge Philosophical Society 126 (1999), no. 2, 369-385.

[7] G. T. Cargo, Angular and tangential limits of Blaschke products and their successive derivatives, Canadian Journal of Mathematics 14 (1962), 334-348.

[8] E. F. Collingwood and A. J. Lohwater, The Theory of Cluster Sets, Cambridge Tracts in Mathematics and Mathematical Physics, no. 56, Cambridge University Press, Cambridge, 1966.

[9] J. J. Donaire, D. Girela, and D. Vukotić, On univalent functions in some Möbius invariant spaces, Journal für die reine und angewandte Mathematik 553 (2002), 43-72.

[10] P. L. Duren, Theory of $H^{p}$ Spaces, Pure and Applied Mathematics, vol. 38, Academic Press, New York, 1970, reprint: Dover, New York, 2000.

[11] P. L. Duren and A. P. Schuster, Bergman Spaces, Mathematical Surveys and Monographs, vol. 100, American Mathematical Society, Rhode Island, 2004.

[12] T. M. Flett, The dual of an inequality of Hardy and Littlewood and some related inequalities, Journal of Mathematical Analysis and Applications 38 (1972), 746-765.

[13] D. Girela and J. Á. Peláez, Non-stable classes of analytic functions, International Journal of Pure and Applied Mathematics 21 (2005), no. 4, 553-563.

[14] _ Carleson measures for spaces of Dirichlet type, to appear in Integral Equations and Operator Theory.

[15] _ Growth properties and sequences of zeros of analytic functions in spaces of Dirichlet type, to appear in Journal of the Australian Mathematical Society.

[16] H. Hedenmalm, B. Korenblum, and K. H. Zhu, Theory of Bergman Spaces, Graduate Texts in Mathematics, vol. 199, Springer, New York, 2000.

[17] J.-P. Kahane and R. Salem, Ensembles parfaits et séries trigonométriques, Actualités Sci. Indust., no. 1301, Hermann, Paris, 1963.

[18] H. O. Kim, Derivatives of Blaschke products, Pacific Journal of Mathematics 114 (1984), no. 1, $175-190$.

[19] J. R. Kinney, Tangential limits of functions of the class $S_{\alpha}$, Proceedings of the American Mathematical Society 14 (1963), 68-70.

[20] J. E. Littlewood, On a theorem of Fatou, Journal of the London Mathematical Society 2 (1927), $172-176$.

[21] J. E. Littlewood and R. E. A. C. Paley, Theorems on Fourier series and power series. II., Proceedings of the London Mathematical Society 42 (1936), 52-89.

[22] A. J. Lohwater and G. Piranian, The boundary behavior of functions analytic in a disk, Annales Academiae Scientiarum Fennicae. Series A I 1957 (1957), no. 239, 17.

[23] A. Nagel, W. Rudin, and J. H. Shapiro, Tangential boundary behavior of functions in Dirichlet-type spaces, Annals of Mathematics. Second Series 116 (1982), no. 2, 331-360.

[24] D. Protas, Blaschke products with derivative in $H^{p}$ and $B^{p}$, The Michigan Mathematical Journal 20 (1973), 393-396.

[25] W. Rudin, The radial variation of analytic functions, Duke Mathematical Journal 22 (1955), no. 2, $235-242$.

[26] J. B. Twomey, Tangential limits for certain classes of analytic functions, Mathematika 36 (1989), no. $1,39-49$.

[27] _ Radial variation of functions in Dirichlet-type spaces, Mathematika 44 (1997), no. 2, 267-277. 


\section{Boundary behaviour}

[28] S. A. Vinogradov, Multiplication and division in the space of analytic functions with an area-integrable derivative, and in some related spaces, Rossiǔ skaya Akademiya Nauk. Sankt-Peterburgskoe Otdelenie. Matematicheskiĭ Institut im. V. A. Steklova. Zapiski Nauchnykh Seminarov (POMI) 222 (1995), no. 23, 45-77, 308, translation in Journal of Mathematical Sciences (New York) 87 (1997), no. 5, 3806-3827, Issled. po Linein. Oper. i Teor. Funktsii.

[29] K. H. Zhu, Analytic Besov spaces, Journal of Mathematical Analysis and Applications 157 (1991), no. 2, 318-336.

[30] A. Zygmund, On certain integrals, Transactions of the American Mathematical Society 55 (1944), no. 2, 170-204.

[31] _ On a theorem of Littlewood, Summa Brasiliensis Mathematicae 2 (1949), no. 5, 51-57.

[32] _ , Trigonometric Series. Vols. I, II, 2nd ed., Cambridge University Press, New York, 1959.

Daniel Girela: Departamento de Análisis Matemático, Facultad de Ciencias, Universidad de Málaga, Campus de Teatinos, 29071 Málaga, Spain

E-mail address: girela@uma.es

José Ángel Peláez: Departamento de Matemática Aplicada, Escuela Politécnica,

Universidad de Málaga, Campus de El Ejido, 29071 Málaga, Spain

E-mail address: pelaez@anamat.cie.uma.es 